\title{
Acne Vulgaris Successfully Treated with Long-Pulsed 1,064- nm Neodymium:Yttrium-Aluminum-Garnet Laser
}

\author{
Hae-Jin Lee ${ }^{1,2}$ \\ Yun Jin $\mathrm{Kim}^{3}$ \\ Sang Joo Lee ${ }^{4}$ \\ Chung Hyeok Lee ${ }^{2}$ \\ Eung $\mathrm{Ho} \mathrm{Choi}^{2}$ \\ Seung Hun Lee ${ }^{1}$ \\ Young Koo Kim ${ }^{4}$
}

${ }^{1}$ Yonsei A\&B Clinic of Dermatology, Seoul, Korea ${ }^{2}$ Department of Dermatology, Yonsei University Wonju College of Medicine, Wonju, Korea ${ }^{3}$ Pureen Dermatologic Clinic, Seoul, Korea ${ }^{4}$ Yonsei Star Skin \& Laser Clinic, Seoul, Korea
Received December 2, 2018

Accepted December 6, 2018

\section{Correspondence}

Young Koo Kim

Yonsei Star Skin \& Laser Clinic, 73 Sinchon-ro,

Seodaemun-gu, Seoul 03789, Korea

Tel.: +82-33-741-1384

Fax: +82-33-748-2650

E-mail: yonseistar64ahanmail.net

(C) Korean Society for Laser Medicine and Surgery

(c) This is an open access article distributed under the terms of the Creative Commons Attribution NonCommercial License (http://creativecommons.org/ licenses/by-nc/4.0) which permits unrestricted noncommercial use, distribution, and reproduction in any medium, provided the original work is properly cited.
Acne vulgaris is a chronic skin disease of pilosebaceous units with multifactorial pathogenesis that affects about $80 \%$ of adolescents and young adults. Although various treatment modalities for acne are reported, treatment of acne with minimal side effects is challenging. In this report, we treated a patient with acne vulgaris using long-pulsed 1,064-nm neodymium:yttrium-aluminum-garnet (Nd:YAG) lasers. After two sessions of long-pulsed 1,064-nm Nd:YAG laser treatment, the patient presented with nearly complete improvement with no remarkable side effects or recurrence over the duration of 6 months. We suggest that long-pulsed 1,064-nm Nd:YAG laser can be effectively and safely used to treat inflammatory lesions in the skin, particularly acne vulgaris, in Asian patients.

\section{Key words}

Acne vulgaris; Long-pulsed 1,064 nm; Neodymium:yttrium-aluminumgarnet laser 


\section{INTRODUCTION}

Acne vulgaris is one of the most common chronic skin diseases of pilosebaceous units resulting from androgeninduced increased sebum production, altered keratinization, inflammation, and bacterial colonization of hair follicles on the face, neck, chest, and back by Propionibacterium acnes. ${ }^{1}$ It affects approximately $80 \%$ of adolescents and young adults. ${ }^{2}$ Acne pathogenesis is generally associated with excessive follicular keratinization, androgen related hyperplasia of the sebaceous glands and increased sebum, colonization with Propionibacterium acnes and inflammatory response including expression of protease-activated receptor-2 leading to epidermal barrier disruption. ${ }^{3}$

Among various treatment modalities for acne vulgaris, lasers may be a good alternative therapeutic options for those patients who fail to respond to or could not tolerate other acne treatments. Various reports of treatment modalities using in patients of acne vulgaris with different light sources, has been detected recently. ${ }^{1,2}$ Nevertheless, the anti-acne mechanisms associated with some lasers including remain largely unknown and need to be elucidated.

Herein, we report a case of acne vulgaris using longpulsed 1,064-nm neodymium:yttrium-aluminum-garnet (Nd:YAG) lasers. After monthly two sessions of longpulsed 1,064-nm Nd:YAG laser treatment, the patient presented nearly complete improvement in the lesions with no remarkable side effects or recurrence over the duration of 6 months.

\section{CASE REPORT}

A 19-year-old, Korean woman visited our clinic with multiple erythematous papules with pustules on the face and neck (Fig. 1). The patient was clinically diagnosed with acne vulgaris (Fig. 1A, 1C) and was scheduled to undergo laser treatment. After obtaining written informed consent, the patient was treated with 2 sessions monthly of long-pulsed 1,064-nm Nd:YAG laser treatment (GentleYAG ${ }^{T M}$; Syneron-Candela Inc., Irvine, CA, USA) and was performed at the combination treatment with painting motion technique $110-\mathrm{mm}$ spot size, $13 \mathrm{~J} / \mathrm{cm}^{2}$ fluence with 0.3 milliseconds (ms) of exposure time), and a 10 $\mathrm{mm}$ spot size, $30 \mathrm{~J} / \mathrm{cm}^{2}$ fluence with $30 \mathrm{~ms}$ of exposure time, and a total of approximately 200 shots on the skin lesion. ${ }^{4}$ The treated areas were cooled with dynamic cooling device (DCD) spray (pre $30 \mathrm{~ms}$ post $30 \mathrm{~ms}$ delay 30 $\mathrm{ms})$, and no prophylactic systemic or topical corticosteroids were prescribed. The patient was recommended to apply nadifloxacin cream and moisturizers. At 6 months after the first treatment of long-pulsed 1,064-nm Nd:YAG laser treatment, the patient presented nearly complete improvement of the acne lesions without worsening or recurrence (Fig. 1B, 1D). After the treatment, the patient also wanted to treat of folliculitis on her chest. The same treatment session was performed and the lesion on her
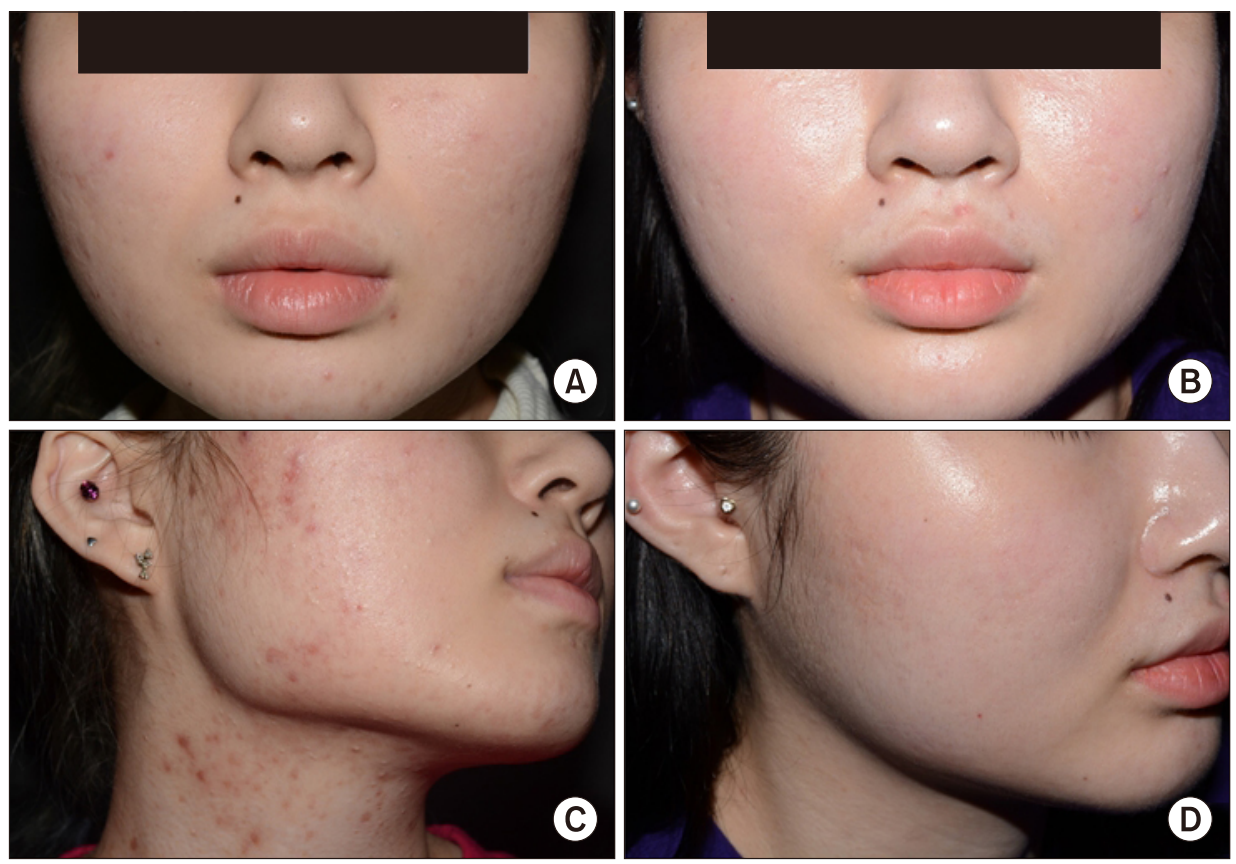

Fig. 1. Photographs of a 19-year-old, Koream female with acne vulgaris $(\mathrm{A}, \mathrm{C})$ at baseline, $(\mathrm{B}, \mathrm{D})$ after 2 sessions of long-pulsed 1,064-nm neodymium:yttrium-aluminum-garnet laser treatment (6 months after baseline). 
chest also presented nearly complete improvement (data not shown).

\section{DISCUSSION}

Laser treatments using various wavelengths were regarded in response to a need of alternative treatment options for acne. They are based on two mechanisms of action; destruction of $P$. acnes and sebaceous glands. Photodynamic therapy with blue to red light as well as intense pulsed light (IPL) destroy P. acnes by absorption of endogenous porphyrins produced by $P$. acnes resulting in a cytotoxic effect on the bacteria. ${ }^{5}$ In contrast, laser therapy using the near infrared spectrum of light are based on thermal damaging the sebaceous glands. ${ }^{6}$

Althuogh there are numerous studies indicating the effective use of 1,064-nm Nd:YAG lasers for the acne scar treatment, there is very little known about efficacy against acne infection. ${ }^{7}$ The 1,064-nm Nd:YAG laser can penetrate the dermis deeply and cause diffuse heating of dermal tissues without damaging the epidermis. The 1,064-nm $\mathrm{Nd}: Y A G$ lasers have been reported to be effective in the treatment of acne scars and photorejuvenation. ${ }^{2}$ However, the efficacy of long-pulsed 1,064-nm Nd:YAG lasers on inflammatory and noninflammatory lesions of acne vulgaris have barely reported. ${ }^{1}$

The possible mechanism of long-pulsed 1,064-nm $\mathrm{Nd}$ :YAG laser is derived from its aptitude to penetrate into the skin deeply to thermally and selectively destroy overactive sebaceous glands and reduce inflammatory lesion in acne. ${ }^{8}$ Along with its thermal penetration effects, the 1,064-nm Nd:YAG also promotes the healing process and collagen remodeling, which is associated with the increased expression of transforming growth factor $-\beta$ in the skin. ${ }^{9}$

Jung et al. ${ }^{2}$ reported a randomized controlled trial with a dual mode of quasi-long pulse and Q-switched 1,064$\mathrm{nm}$ Nd:YAG laser assisted with a topically applied carbon suspension in the patients with acne vulgaris. The carbon particles are distributed to the stratum corneum, and some of them also penetrated into the hair follicles. When activated by the laser light, the carbon undergoes a rapid temperature increase, thereby heating and damaging the follicles and surrounding structures such as sebaceous gland. Based on the study of Jung et al., ${ }^{2}$ the energy of laser with relatively shorter pulse duration difficultly penetrates the epidermis deeply without carbon suspension. Therefore, laser with longer pulse duration might easily affect sebaceous glands, thereby inflammatory lesion of acne vulgaris could be treated without carbon suspension
In conclusion, the authors suggest that long-pulsed 1,064-nm Nd:YAG lasers can be effectively and safely used for treating inflammatory lesions in the dermis and epidermis, particularly acne vulgaris, in Asian patients. Further prospective, controlled investigations are needed to be followed to compare the efficacy and safety of longpulsed lasers and to optimize the treatment settings for acne vulgaris.

\section{CONFLICT OF INTEREST}

The authors have no conflict of interest to disclose.

\section{REFERENCES}

1. Mohamed EE, Tawfik K, Elsaie M. Intense pulsed light versus 1,064 long-pulsed neodymium: yttrium-aluminum-garnet laser in the treatment of facial acne vulgaris. J Clin Diagn Res 2016;10:WC01-3.

2. Jung JY, Hong JS, Ahn CH, Yoon JY, Kwon HH, Suh DH. Prospective randomized controlled clinical and histopathological study of acne vulgaris treated with dual mode of quasi-long pulse and Q-switched 1064-nm Nd:YAG laser assisted with a topically applied carbon suspension. J Am Acad Dermatol 2012;66:626-33.

3. Lee SE, Kim JM, Jeong SK, Choi EH, Zouboulis CC, Lee SH. Expression of protease-activated receptor-2 in SZ95 sebocytes and its role in sebaceous lipogenesis, inflammation, and innate immunity. J Invest Dermatol 2015;135:2338.

4. Chiba C, Usui A, Hara H, Ishi Y. Clinical experience in skin rejuvenation treatment in Asians using a long-pulse Nd:YAG laser. J Cosmet Laser Ther 2009;11:134-8.

5. Papageorgiou P, Katsambas A, Chu A. Phototherapy with blue $(415 \mathrm{~nm})$ and red $(660 \mathrm{~nm})$ light in the treatment of acne vulgaris. Br J Dermatol 2000;142:973-8.

6. Lloyd JR, Mirkov M. Selective photothermolysis of the sebaceous glands for acne treatment. Lasers Surg Med 2002;31:115-20.

7. Lee DH, Choi YS, Min SU, Yoon MY, Suh DH. Comparison of a 585-nm pulsed dye laser and a 1064-nm Nd:YAG laser for the treatment of acne scars: a randomized split-face clinical study. J Am Acad Dermatol 2009;60:801-7.

8. Bencini PL, Luci A, Galimberti M, Ferranti G. Long-term epilation with long-pulsed neodimium:YAG laser. Dermatol Surg 1999;25:175-8.

9. Lee YB, Kang NH, Eun YS, Cheon MS, Kim KM, Cho BK, et al. Effects of long-pulsed 1,064-nm neodymium-doped yttrium aluminum garnet laser on dermal collagen remodeling in hairless mice. Dermatol Surg 2012;38:985-92. 